\title{
Evaluación de la capacidad para estar en juicio y el uso de técnicas de información y comunicación remotas en la Justicia argentina
} Assessment of competency to stand a trial and the use of remote information and communication techniques in the argentine Justice

\section{Pablo Alejandro Burgueño'}

\section{Resumen}

La pandemia del COVID-19 cambió la forma de trabajo de los médicos forenses, cobrando relevancia en ella el uso de las Tecnologías de la Información y Comunicación (TICs). Pero su implementación para evaluaciones psiquiátrico-forenses debería cumplir con estándares avalados por la comunidad científica. Son necesarios instrumentos psicojurídicos para poder determinar en un imputado la capacidad procesal para estar en juicio penal. En la Justicia de la Ciudad Autónoma de Buenos Aires (CABA) se llevan a cabo actuaciones virtuales, a partir de la declaración del Aislamiento Social Preventivo y Obligatorio (ASPO) decretado por el Gobierno Nacional. En el caso del fuero penal se utilizan plataformas Web y notificaciones electrónicas para el trámite de las causas judiciales. Sin embargo, en la práctica de la medicina legal se advierten dificultades en la utilización de las TICs, para poder determinar la capacidad procesal penal de una persona.

Palabras clave: Evaluación forense - Capacidad para estar en juicio - TICs - Administración de Justicia en Argentina - ECST-R.

\section{Abstract}

The COVID-I 9 has changed the way of working of forensic doctors, and the use of Information and Communication Technologies (ICTs) has gained relevance. But its implementation, for psychiatric-forensic evaluations, should comply with standards endorsed by the scientific community. Psycho-legal instruments are necessary in order to determine the procedural capacity of an accused person to stand trial. In the Justice of the Autonomous City of Buenos Aires (CABA), virtual proceedings are carried out, as the National Government decreed the obligatory social lockdown. In the case of the criminal jurisdiction, web platforms and electronic notifications are used for the processing of legal cases. However, in the practice of forensic medicine, there are difficulties in the use of ICTs to determine a person's capacity for criminal prosecution.

Keywords: Forensic assessment - Capacity to stand trial - ICTS - Administration of Justice in Argentina - ECST-R.

RECIBIDO 2/2/202I - ACEPTADO I/6/202I

1. Médico Forense del Poder Judicial de la Ciudad Autónoma de Buenos Aires, Argentina.

Correspondencia:

pburgueno@jusbaires.gob.ar 


\section{Introducción}

La psiquiatría forense, no se encuentra exenta a los nuevos desafíos que ha presentado el COVID-19. La virtualidad en las evaluaciones psiquiátricas forenses, a través de las TICs, prometen ser una solución, pero aún, se encuentran en una fase temprana de desarrollo.

La ausencia o fallas de la tecnología repercuten en la aplicación de la fina tarea semiológica que se despliega durante los exámenes psicopatológicos presenciales. En ese sentido, se hace necesario repensar la labor a partir de la utilización de las TICs, desarrollando técnicas fiables, con sustento científico para dictaminar la capacidad procesal de los imputados.

\section{Capacidad para estar en juicio y su eva- luación}

En el debido proceso de justicia se requiere que el imputado de un delito tenga capacidad de ejercer su propia defensa, solo o con sus abogados.

La Justicia Penal de la Ciudad Autónoma de Buenos Aires (CABA), en el art. 35 de su código procesal (CPPCABA) (República Argentina. Ley N².303/07, Art. 35) obliga a determinar, al inicio del proceso judicial, la capacidad de autodefensa de un imputado. Si éste se encuentra incapacitado, debe suspenderse el proceso (art. 34) (República Argentina. Ley $\mathrm{N}^{\circ}$ 2.303/07, Art. 34) o se cierran las actuaciones. Por ello, los dictámenes periciales deben ser fundamentados científicamente para asegurar el debido proceso.

La mayoría de los Códigos procesales penales, presuponen que todas las personas son capaces de actuar en su defensa. Por ejemplo, en el fuero Civil, se considera que todas las personas son competentes para llevar a cabo actos jurídicos hasta que se demuestre lo contrario y esto sea declarado por resolución judicial (República Argentina. Código Civil y Comercial de la Nación, 2014).

El método psicopatológico forense, incluye variables de análisis como la observación conductual del examinado, su interrelación con los otros, su capacidad vincular, su adaptación a la realidad, su procesamiento de información, etc. Se analizan así, la postura corporal, los movimientos, las inflexiones de voz, la sudoración ante el estrés, etc.; sin embargo, todo ello escapa al examen forense virtual. Además, no es posible divulgar lo expresado en el espacio pericial. Entonces éstas TICs, deberían garantizar la reserva, las condiciones del lugar de evaluación, determinar quienes estarán presentes, o quien podrá escuchar o grabar lo dicho (Gardiner G, 2020). También debemos sumar los distractores del examinado y del examinador, máxime en la relación virtual (Brodsky, 2019).
En la medicina legal la implementación de las TICs presenta dificultades que podrían inducir un error pericial que modificaría peligrosamente el rumbo procesal. Sin embargo, hay trabajos, que avalan los beneficios de las TICs a nivel forense (Bari et al., 2018). La presencia "virtual" del médico en distintas instancias judiciales evitaría, por ejemplo, el tiempo de espera en la declaración judicial, pudiendo éste realizar otros exámenes u otras tareas mientras tanto.

Pero las peritaciones psicofísicas mediante TICs no reúnen, aún, bases científicas para la búsqueda de la verdad jurídica. La determinación de la capacidad procesal se establece si existe o no un trastorno mental que incapacite la autodefensa. Un informe realizado desde la virtualidad sólo podrá considerarse como un estado básico psicofísico del examinado en un momento dado, no pudiendo abarcar el análisis de una psicosemiología forense completa y menos aún el funcionamiento del psiquismo en un momento pretérito, que auxiliaría para la determinación de la inimputabilidad. Ya se ha estudiado que cuando se quieren expresar emociones y actitudes, un 7\% se hace en forma verbal (lo que se dice), un $38 \%$ a través de lo paraverbal (el cómo se dice) y el 55\% restante, se comunica con lo no verbal. Todo éste material de análisis, escapa a la observación del entrevistador que utiliza las TICs y no la entrevista presencial (Villa Marín López, 2014).

Esta situación ha estimulado el desarrollo de instrumentos para las evaluaciones psicopatológicas con técnicas de investigación y evaluación desde la virtualidad (PARIConnect). Por ejemplo, la Escala de Evaluación de la Competencia para estar en Juicio (versión revisada-ECST-R) traducción del inglés de la Evaluation of Competency to Stand Trial - Revised (ECST-R). Este instrumento, basado en el criterio legal estadounidense llamado "Dusky standard", fue desarrollado en 2004 por Rogers, Tillbrook y Sewell (Rogers et al., 2004; Stafford \& Sellbom, 2012), y evalúa el funcionamiento del estado psíquico de una persona, apuntando a las bases fundamentales que conforman la capacidad procesal, es decir, el comprender los hechos imputados, la capacidad de relación con su defensa y ciertas capacidades como la inteligencia, la cognición y la voluntad (Zapf \& Viljoen, 2003; Maier, 2003). La prueba consiste en una entrevista con una parte semiestructurada y otra estructurada, de 18 elementos divididos en 4 escalas: a.Comprensión de los hechos que tienen lugar en los procedimientos judiciales, $\mathbf{b}$. Entendimiento racional de los procedimientos judiciales, c. Capacidad de consultar con un abogado y d. Habilidad racional general; e incluye también 28 ítems 
adicionales divididos en 5 escalas que evalúan estilo de respuesta (Murrie \& Zelle, 2015).

La ECST-R, adaptada al español (Montero \& Fernández-Pinto, 2013) se encuentra en proceso de validación para ser utilizada en la Justicia de la CABA y permitiría realizar evaluaciones fiables con rigor científico.

La aplicación de la escala no reemplazaría el examen presencial, pero permitiría evaluar la capacidad procesal, a través de una entrevista virtual estructurada, formando parte de las herramientas diagnósticas específicas.

\section{Una frustrada prueba desde lo virtual}

En la actualidad, debido a los protocolos de distanciamiento físico impuestos por la pandemia, las evaluaciones médico forenses presenciales en la Justicia de la CABA son excepcionales y han sido reemplazadas por el uso de TICs. En abril de 2020, se realizó una "tele-peritación" en una plataforma Web, adonde intervinieron el imputado (detenido en un penal), una psiquiatra y una psicóloga del servicio de Salud Mental del mismo penal, los peritos oficiales y los peritos de la defensa (De Marco Calzada, 2020).

Los puntos periciales consistían en evaluar la capacidad procesal del imputado y la capacidad de comprensión de la criminalidad de su accionar y dirigir su conducta al momento del injusto.

El Juez determinó que el examen fuera realizado por el personal de Salud Mental del penal y que el resto de los peritos oficiaran, únicamente, como controladores de la prueba.

Los profesionales de salud penitenciarios eran especialistas en lo asistencial, pero no en lo legal. Por tanto, solo se logró determinar, en ese momento, el estado psíquico del imputado.

No se utilizó ningún instrumento psicojurídico específico y validado científicamente, no pudiendo aplicarse la fina tarea de la psicosemiología, en forma remota. Con estas limitaciones, el valor jerárquico de la prueba resultó casi nulo, finalizando como un dato más y no como una opinión de los expertos, fundamentada en bases científicas.

Se advierten dos cuestiones: por un lado, como un aspecto positivo, la posible utilización de las TICs como complemento del examen presencial, y por otro lado, la necesidad de contar con un instrumento estandarizado. La aplicación de la escala ECST-R, en forma dirigida y estructurada, evaluando las capacidades intelectivas, cognitivas y volitivas para tener suficiente capacidad procesal, permitiría combinar los recursos y asegurar análisis fiables de las capacidades procesales de los imputados.

\section{Conclusiones}

La pandemia ha modificado las formas de pensar las interacciones sociales y ha permitido la revisión sistemática de sus prácticas. En nuestro caso, la incorporación de las TICs a la praxis garantizó la aplicación de justicia. Sin embargo, la utilización de estas técnicas debe sustentarse en los principios de fiabilidad, validez y confidencialidad de la información obtenida, debiéndose utilizarlas con ética profesional.

La utilización de la escala ECST-R, no invalidaría el uso de las TICs, pero su aplicación garantizaría resultados fiables, reforzando la jerarquización de la prueba pericial.

Conflictos de intereses: El autor declara no tener conflicto de intereses.

\section{Referencias bibliográficas}

Bari, S., Arora, P., Gupta, A. K., Singh, M., Aggarwal, A. K. (2018). Tele-evidence: A videoconferencing tool as a viable alternative to physical appearance of doctors for the judicial summons. Year, 64(4), 206-211.

Brodsky, S. L. (2019). Distractions in Forensic Evaluations. Journal of the American Academy of Psychiatry and the Law, Online May, JAAPL.003842-19. doi: https://doi.org/10.29158/JAAPL.003842-19

De Marco Calzada, G. (2020). Perito Psicóloga del Ministerio Público de la Defensa, Justicia de la Ciudad Autónoma de Buenos Aires. Comunicación personal.

Gardiner G, Perito Psicóloga, Asesora Técnica de SCJBA, documento de trabajo, La Plata, 29 de marzo de 2020.

Maier J. B. J. (2003). Derecho Procesal penal, Tomo II Parte general, Sujetos Procesales, $1^{\circ}$ edición, Edit. Del Puerto.

Montero, D., Fernández-Pinto, I. (2013). Sistema para la evaluación de la conducta adaptativa (ABAS-II). Dpto. TEA Ediciones.

Murrie, D. \& Zelle, H. (2015). Criminal competencies. En: APA Handbook of Forensic Psychology, 1, 115-157.

PARIConect. https://www.parinc.com/What-is-PARiConnect Último acceso: 12 de noviembre de 2020.

Stafford, K. D. \& Shellbom, M. (2013). Assesment of Competence to Stand Trial. En: Handbook of Psychology: Forensic psychology, (pp 412-439).

República Argentina. "Código Procesal Penal de la Ciudad Autónoma de Buenos Aires”. Ley N².303/07, Art. 34 http://www.cedom.gov.ar/codigos.aspx.

República Argentina. "Código Procesal Penal de la Ciudad Autónoma de Buenos Aires”. Ley N².303/07, Art. 35. http://www.cedom.gov.ar/codigos.aspx. Último acceso: 15 de noviembre de 2020.

República Argentina. Código Civil y Comercial de la Nación, Ley 26994. Decreto 1795/2014, Art. 22. http://servicios.infoleg.gob.ar/infolegInternet/anexos/235000-239999/235975/texact.htm\#6; Último acceso: 15 de noviembre de 2020 .

Rogers, R., Tillbrook, C. E., y Sewell, K. W. (2004). Evaluation of Competency to Stand Trial-Revised (ECST R). Psyhological Assessment Resources.

Villa Marín López M, (2014). Neurociencia y detección de la verdad y del engaño en el proceso penal (p. 25). Edit. Marcial Pons.

Zapf, P. A., \& Viljoen, J. L. (2003). Issues and considerations regarding the use of assessment instruments in the evaluation of competency to stand trial. Behavioral Sciences \& the Law, 21(3), 351-367. doi:10.1002/bsl.535 